\section{Dupilumab improves nasal polyp burden and asthma control in patients with CRSwNP and AERD}

Tanya M. Laidlaw, MD ${ }^{a}$, Joaquim Mullol, MD ${ }^{b}$, Chunpeng Fan, $\mathrm{PhD}^{\mathrm{c}}$, Donghui Zhang, $\mathrm{PhD}^{\mathrm{c}}$, Nikhil Amin, MD ${ }^{d}$, Asif Khan, MBBS, $\mathrm{MPH}^{\mathrm{e}}$, Jingdong Chao, $\mathrm{PhD}^{\mathrm{d}}$, and Leda P. Mannent, $\mathrm{MD}^{\mathrm{e}}$

Clinical Implications

- In the difficult-to-treat subgroup of patients with chronic rhinosinusitis with nasal polyps (CRSwNP) and comorbid aspirin-exacerbated respiratory disease, dupilumab significantly improved CRSwNP disease outcomes, along with asthma control and lung function. This is preliminary evidence of the effect of dupilumab in patients with CRSwNP and comorbid aspirinexacerbated respiratory disease.

\section{TO THE EDITOR:}

Chronic rhinosinusitis with nasal polyps (CRSwNP) is a predominantly type $2-$ mediated inflammatory disease associated with a high symptom burden and poor health-related quality of life. ${ }^{1}$ Dupilumab, a fully human VelocImmune-derived ${ }^{2,3} \mathrm{mAb}$, blocks the shared receptor component for IL-4 and IL-13, thus inhibiting signaling of both IL-4 and IL-13, cytokines that are key drivers of type 2 diseases such as atopic dermatitis (AD), asthma, allergic rhinitis, and food allergies, which are often associated as comorbidities. Dupilumab is approved by the US Food and Drug Administration for the treatment of adults with moderate-to-severe $\mathrm{AD}$ whose disease is inadequately controlled with topical prescription therapies or for whom those therapies are not advisable, and can be used with or without topical corticosteroids, and by the European Medicines Agency for use in adults with moderate-to-severe $\mathrm{AD}$ who are candidates for systemic therapy. Dupilumab is also approved by the US Food and Drug Administration as an add-on maintenance treatment in patients 12 years or older with moderate-tosevere asthma with an eosinophilic phenotype or with oral corticosteroid-dependent asthma, ${ }^{4}$ and has demonstrated positive results in proof-of-concept studies for patients with eosinophilic esophagitis. ${ }^{5}$

Bachert et $\mathrm{al}^{6}$ have described a phase $2 \mathrm{a}$ dupilumab trial in patients with CRSwNP refractory to intranasal corticosteroids. In this proof-of-concept study (ClinicalTrials.gov Identifier: NCT01920893), dupilumab in conjunction with mometasone furoate nasal spray significantly improved endoscopic, radiographic, clinical, and patient-reported outcomes in these patients, many of whom had comorbid conditions. Patients with CRSwNP with comorbid aspirin-exacerbated respiratory disease (AERD), also referred to as nonsteroidal anti-inflammatory drugexacerbated respiratory disease, are among the most severe and difficult to treat. ${ }^{7}$ An estimated $8 \%$ to $26 \%$ of patients with CRSwNP are reported to have comorbid AERD. ${ }^{8,9}$
Of the 60 patients with CRSwNP enrolled in the NCT01920893 study, 19 (31.7\%) had self-reported comorbid AERD ( 8 in the dupilumab-treated group and 11 in the placebo group); this included patients reporting sensitivity to aspirin as well as other nonsteroidal anti-inflammatory drugs. Most patients reporting AERD were women (57.9\%), and there was a much lower proportion of women in the aspirin-tolerant subgroup (36.6\%). At baseline, the patients with AERD had a significantly higher mean Lund-Mackay total score than did patients without AERD (21.2 \pm 2.4 vs $17.5 \pm 5.8$, respectively; $P=.03)$. In patients with comorbid asthma, mean $\mathrm{FEV}_{1}(\mathrm{~L})$ was lower, although not significantly so, in those with AERD compared with those who were aspirin-tolerant ( $2.4 \pm 0.6$ vs 2.9 \pm 0.9 , respectively). Patients with AERD experienced severe loss of smell at baseline, more so than aspirin-tolerant patients, as indicated by the higher mean score for the "sense of smell/taste" question in the 22-item Sino-Nasal Outcome Test (SNOT-22) and the lower mean score for the University of Pennsylvania Smell Identification Test in the former group ( $4.7 \pm 0.6$ vs 4.2 \pm 1.2 and $11.2 \pm 4.9$ vs $15.6 \pm 9.0$, respectively; $P=$ not significant for both). Baseline data are presented in Table E1 in this article's Online Repository at www.jaci-inpractice.org.

Mean baseline nasal polyp score (NPS) was similar among patients with and without AERD (a score of $\geq 5$ was required for participation in the study). ${ }^{6}$ Dupilumab treatment resulted in a significant reduction in mean NPS from baseline to week 16 in the AERD patient subgroup (least-squares mean difference vs placebo of -2.51). Aspirin-tolerant patients also showed reductions in NPS (least-squares mean difference vs placebo of -0.72), but these did not reach statistical significance (Figure 1, A). At week 16, dupilumab-treated patients in both the AERD and aspirin-tolerant subgroups experienced significant improvements in Lund-Mackay total score, SNOT-22 total score, SNOT-22 sense of smell/taste score, and University of Pennsylvania Smell Identification Test score, and significant reductions in daily morning nasal congestion/obstruction score (Figure 1, B, $C, D, E$, and $F$, respectively) compared with placebo. Dupilumab treatment significantly improved the 5-item Asthma Control Questionnaire total score in patients with AERD and comorbid asthma at weeks 12 and 16 vs placebo. In aspirin-tolerant patients with comorbid asthma, the change from baseline with dupilumab treatment versus placebo was significant at weeks 8, 12, and 16 (Figure 2, $A$ and $B$ ). In dupilumab-treated patients with AERD and comorbid asthma, there was a significant change from baseline in $\mathrm{FEV}_{1}$ (L) at weeks 8 and 16 $(380 \mathrm{~mL}$ and $360 \mathrm{~mL}$, respectively) compared with placebo group patients. Changes were clinically meaningful at all time points, ranging from $160 \mathrm{~mL}$ to $380 \mathrm{~mL}$. Although not significant, the change from baseline $\mathrm{FEV}_{1}(\mathrm{~L})$ in aspirin-tolerant patients with comorbid asthma, dupilumab versus placebo, was clinically meaningful at all time points, ranging from $150 \mathrm{~mL}$ to $210 \mathrm{~mL}$ (statistical nonsignificance was most likely due to the small sample size) (Figure 2, $C$ and $D$ ).

Treatment-emergent adverse events (TEAEs) were similar among patient subgroups based on the presence/absence of AERD comorbidity. Of the TEAEs that occurred in more than $10 \%$ of either the dupilumab or placebo treatment group, 


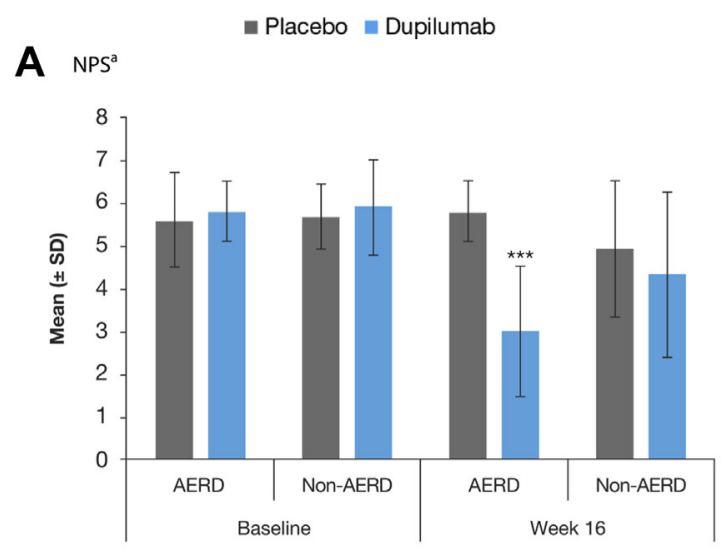

B LMK total score ${ }^{\mathrm{b}}$

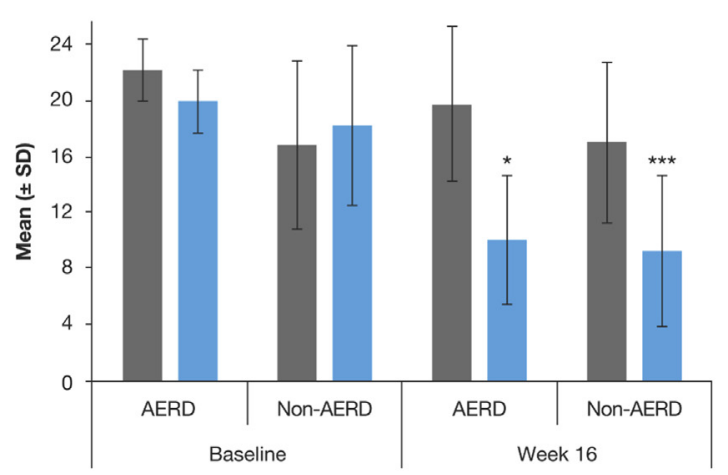

C SNOT-22 total score ${ }^{c}$

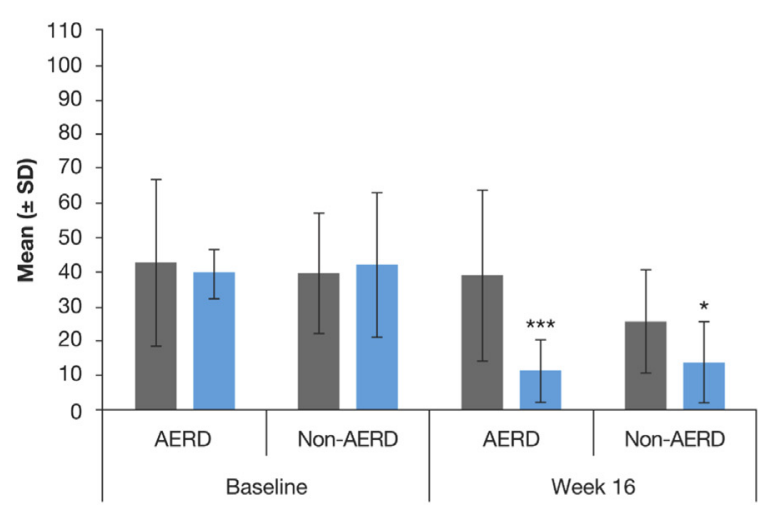

D sNOT-22 sense of smell/taste score ${ }^{d}$

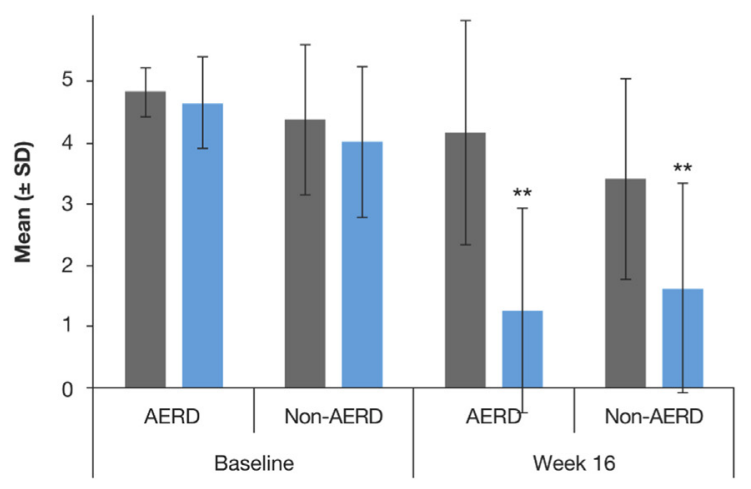

E UPSIT score

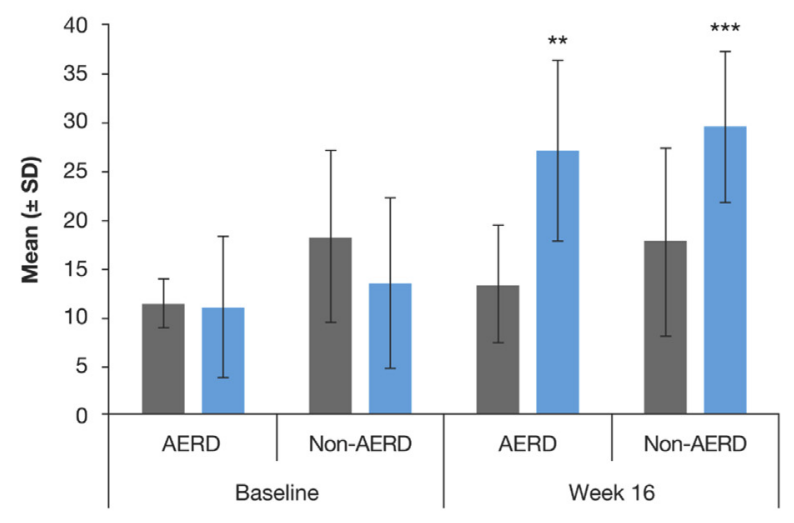

F AM nasal congestion/obstruction scoré

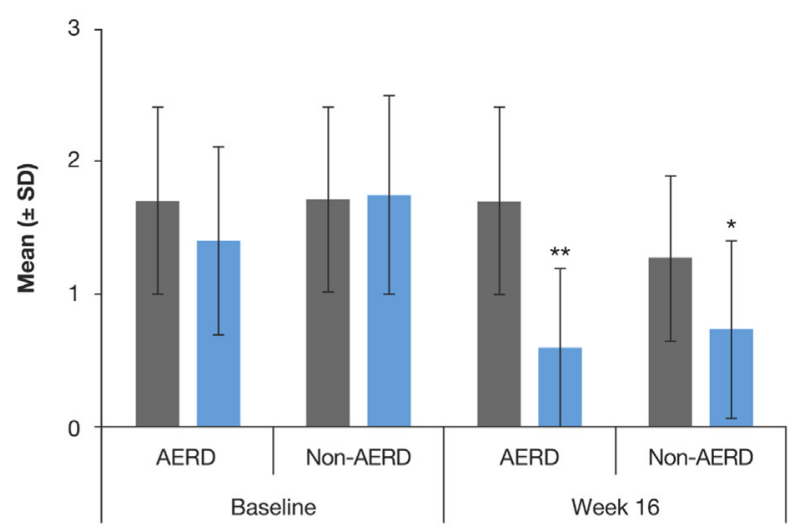

FIGURE 1. Disease burden/symptoms at baseline and at week 16 by (A) NPS, (B) LMK total score, (C) SNOT-22 total score, (D) SNOT-22 sense of smell/taste score, (E) UPSIT score, and (F) AM nasal congestion/obstruction score. AM, Morning; LMK, Lund-Mackay; UPSIT, University of Pennsylvania Smell Identification Test. AERD: placebo $n=11$, dupilumab $n=8$. Non-AERD: placebo $n=19$, dupilumab $\mathrm{n}=22$. Non-AERD represents aspirin-tolerant patients. In Figure $1, A$, range of 0 to 8 ; higher scores indicate worse polyp burden. In Figure $1, B$, range of 0 to 24; higher scores indicate more opacification. In Figure 1, $C$, range of 0 to 110 ; higher scores indicate poorer outcomes, and differences greater or equal to 8.9 are considered clinically meaningful. In Figure $1, D$, range of 0 to 5 ; higher scores indicate worse sense of smell. In Figure 1,E, range of 0 to 40; higher scores indicate better sense of smell, scores of 35 to 40 indicate normal sense of smell. In Figure 1, $F$, range of 0 to 3 ; symptoms were captured using a categorical scale $(0=$ no symptoms, $1=$ mild, $2=$ moderate, $3=$ severe). ${ }^{*} P<.05,{ }^{*} P<.005,{ }^{*}{ }^{*} P<.0005$. $P$ value represents change from baseline, dupilumab vs placebo. 
A ACQ-5 score at baseline and at Week 16

\section{- Placebo = Dupilumab}

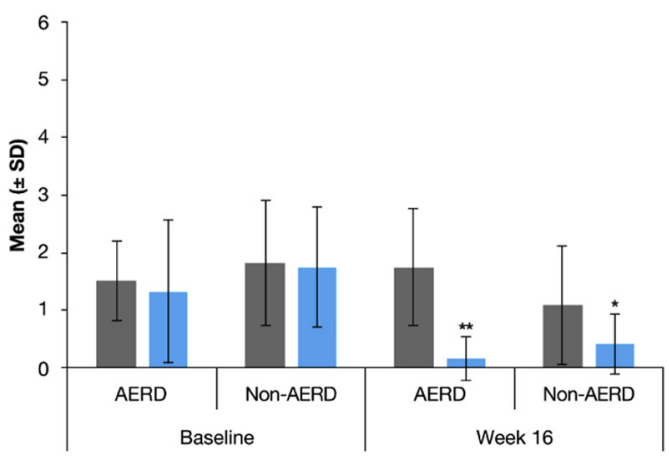

B

$A C Q-5$ score ${ }^{a}$ by visit

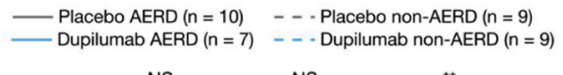
$\begin{array}{llll} & N S & \text { NS } & * \\ * & N S & * & *\end{array}$

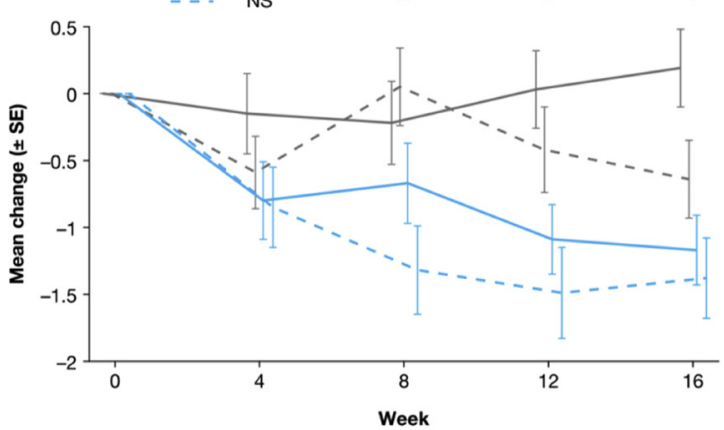

C $\mathrm{FEV}_{1}(\mathrm{~L})$ at baseline and at Week 16

- Placebo = Dupilumab

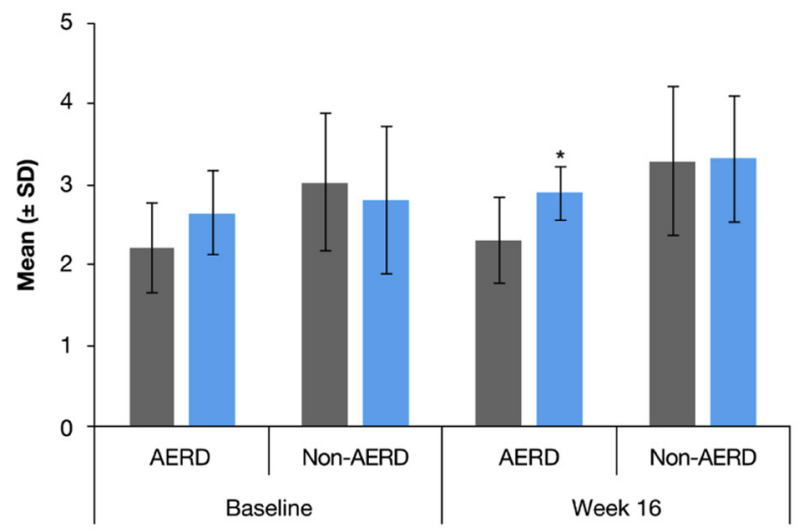

D $\mathrm{FEV}_{1}(\mathrm{~L})$ by visit

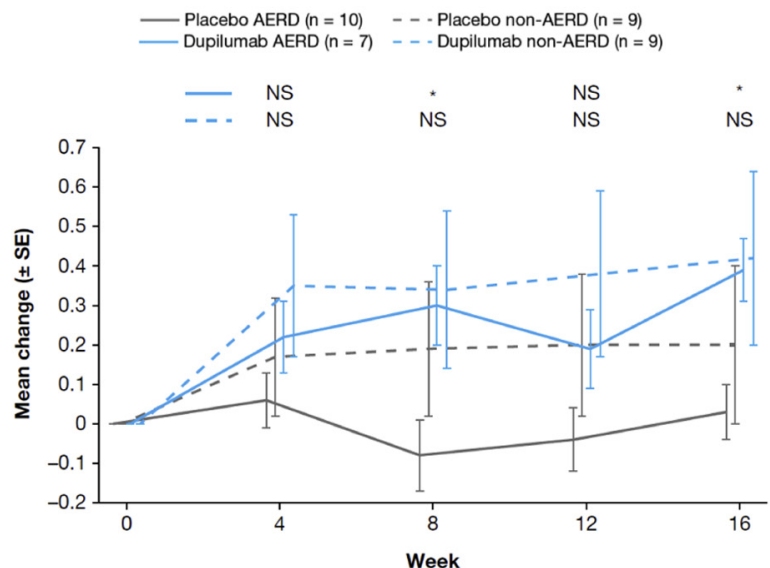

FIGURE 2. Asthma-related outcomes, including ACQ-5 score (A) at baseline and at week 16 and (B) by visit, and (C) FEV 1 (L) at baseline and at week 16 and (D) by visit for AERD patient subgroups with comorbid asthma. ACQ-5, 5-Item Asthma Control Questionnaire; NS, not significant. AERD with asthma: placebo $\mathrm{n}=10$, dupilumab $\mathrm{n}=7$. Non-AERD with asthma: placebo $\mathrm{n}=9$, dupilumab $\mathrm{n}=9$. Non-AERD represents aspirin-tolerant patients. ACQ-5 score: Range of 0 to 6 ; lower scores indicate better asthma control, and differences greater than or equal to 0.5 are considered clinically meaningful. ${ }^{*} P<.05,{ }^{*} P<.005$. $P$ value represents change from baseline, dupilumab vs placebo.

epistaxis (reported by 8 aspirin-tolerant patients; 1 patient with AERD), oropharyngeal pain (experienced by 8 aspirin-tolerant patients; 1 patient with AERD), and bronchitis (occurring in 5 aspirin-tolerant patients and in 0 patient with AERD) were more common in aspirin-tolerant patients than in those with AERD. TEAEs for the intent-to-treat population have been described previously by Bachert et al.

In summary, patients with AERD had greater sinus opacification and worse sense of smell (a symptom typically associated with CRSwNP, particularly in those with comorbid AERD) at baseline compared with aspirin-tolerant patients with CRSwNP, and patients with CRSwNP with comorbid asthma and AERD had poorer lung function at baseline than did patients with CRSwNP with asthma without AERD. These baseline results indicate more severe disease in patients with AERD. Dupilumab significantly improved CRSwNP disease outcomes, in addition to improving asthma control and lung function, in the study population and, in particular, in the difficult-to-treat subgroup of patients with CRSwNP with comorbid AERD. Although larger studies are needed to confirm efficacy, this is preliminary evidence of the effect of dupilumab in a small number of patients with CRSwNP and comorbid AERD.

\section{Acknowledgments}

We thank Qunming Dong for his contribution.

aBrigham and Women's Hospital and Harvard Medical School, Boston, Mass

bHospital Clínic-IDIBAPS, CIBERES, Universitat de Barcelona, Catalonia, Spain

${ }^{\mathrm{c}}$ Sanofi, Bridgewater, NJ

${ }^{\mathrm{d}}$ Regeneron Pharmaceuticals, Inc, Tarrytown, NY

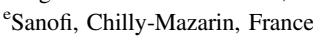

This research was sponsored by Sanofi and Regeneron Pharmaceuticals (ClinicalTrials.gov Identifier: NCT01920893). Medical writing/editorial assistance provided by Sinéad Holland, PhD, of Excerpta Medica, was funded by Sanofi Genzyme and Regeneron Pharmaceuticals. 
Conflicts of interest: T. M. Laidlaw has been a member of national and international scientific advisory boards (consulting) for Allakos, GlaxoSmithKline, SanofiAventis, and Regeneron Pharmaceuticals, Inc. J. Mullol has been a member of national and international scientific advisory boards (consulting) and has received fees for lectures and grants for research projects from ALK-Abelló, Allakos, FAES, Genentech, Glenmark, GlaxoSmithKline, Mylan, Menarini, MSD, Novartis, Regeneron Pharmaceuticals, Inc, Sanofi Genzyme, UCB, and Uriach. C. Fan, D. Zhang, A. Khan, and L. P. Mannent are employed with Sanofi and may hold stock and/or stock options in the company. N. Amin and J. Chao are employed with Regeneron Pharmaceuticals, Inc, and are shareholders.

Received for publication December 27, 2018; revised March 25, 2019; accepted for publication March 28, 2019.

Available online

Corresponding author: Tanya M. Laidlaw, MD, Department of Medicine, Division of Allergy, Immunology and Rheumatology, Brigham and Women's Hospital, 75 Francis St, Boston, MA 02115. E-mail: tlaidlaw@partners.org.

2213-2198

(C) 2019 American Academy of Allergy, Asthma \& Immunology

https://doi.org/10.1016/j.jaip.2019.03.044

\section{REFERENCES}

1. Fokkens W, Lund VJ, Mullol J. European Position Paper on Rhinosinusitis and Nasal Polyps Group. European position paper on rhinosinusitis and nasal polyps 2007. Rhinol Suppl 2007;20:1-136.
2. Macdonald LE, Karow M, Stevens S, Auerbach W, Poueymirou WT, Yasenchak J, et al. Precise and in situ genetic humanization of $6 \mathrm{Mb}$ of mouse immunoglobulin genes. Proc Natl Acad Sci U S A 2014;111:5147-52.

3. Murphy AJ, Macdonald LE, Stevens S, Karow M, Dore AT, Pobursky K, et al. Mice with megabase humanization of their immunoglobulin genes generate antibodies as efficiently as normal mice. Proc Natl Acad Sci U S A 2014;111:5153-8.

4. Castro M, Corren J, Pavord ID, Maspero J, Wenzel S, Rabe KF, et al. Dupilumab efficacy and safety in moderate-to-severe uncontrolled asthma. N Engl J Med 2018;378:2486-96.

5. Hirano I, Dellon ES, Hamilton JD, Collins MH, Peterson K, Chehade M. Dupilumab efficacy and safety in adult patients with active eosinophilic esophagitis: a randomized double-blind placebo-controlled phase 2 trial. Presented at the 2017 annual scientific meeting of the American College of Gastroenterology; October 16, 2017. Orlando, FL.

6. Bachert C, Mannent L, Naclerio RM, Mullol J, Ferguson BJ, Gevaert P, et al. Effect of subcutaneous dupilumab on nasal polyp burden in patients with chronic sinusitis and nasal polyposis: a randomized clinical trial. JAMA 2016;315: 469-79.

7. Kowalski ML, Agache I, Bavbek S, Bakirtas A, Blanca M, Bochenek G, et al. Diagnosis and management of NSAID-exacerbated respiratory disease (N-ERD)—a EAACI position paper. Allergy 2019;74:28-39.

8. Mullol J, Picado C. Rhinosinusitis and nasal polyps in aspirin-exacerbated respiratory disease. Immunol Allergy Clin North Am 2013;33:163-76.

9. Laidlaw TM, Boyce JA. Aspirin-exacerbated respiratory disease-new prime suspects. N Engl J Med 2016;374:484-8. 


\section{ONLINE REPOSITORY}

TABLE E1. Baseline demographic and clinical characteristics

\begin{tabular}{|c|c|c|c|}
\hline Characteristic & AERD ( $n=19)$ & Non-AERD $(n=41)$ & $\begin{array}{l}P \text { value AERD } \\
\text { vs non-AERD }\end{array}$ \\
\hline Age $(y)$, mean \pm SD & $50.5 \pm 5.4$ & $47.4 \pm 10.7$ & NS \\
\hline Sex: female, n $(\%)$ & $11(57.9)$ & $15(36.6)$ & NS \\
\hline Nasal polyposis duration $(y)$, mean $\pm \mathrm{SD}$ & $10.6 \pm 8.0$ & $9.0 \pm 7.6$ & NS \\
\hline Patients with comorbid asthma, n (\%) & $17(89.5)$ & $18(43.9)$ & .001 \\
\hline \multicolumn{4}{|l|}{ Disease burden/symptoms } \\
\hline Endoscopic NPS, mean \pm SD & $5.7 \pm 1.0$ & $5.8 \pm 1.0$ & NS \\
\hline CT scan LMK total score, mean \pm SD & $21.2 \pm 2.4$ & $17.5 \pm 5.8$ & .026 \\
\hline SNOT-22 score, mean \pm SD & $41.3 \pm 18.7$ & $40.9 \pm 19.3$ & NS \\
\hline SNOT-22 sense of smell/taste score, mean \pm SD & $4.7 \pm 0.6$ & $4.2 \pm 1.2$ & NS \\
\hline UPSIT, mean \pm SD & $11.2 \pm 4.9$ & $15.6 \pm 9.0$ & .050 \\
\hline $\mathrm{AM}$ symptom score for nasal congestion/obstruction, mean $\pm \mathrm{SD}$ & $1.6 \pm 0.7$ & $1.7 \pm 0.7$ & NS \\
\hline Asthma-related symptoms & $\mathbf{n}=\mathbf{1 7}$ & $\mathbf{n}=\mathbf{1 8}$ & \\
\hline ACQ-5 score, mean \pm SD & $1.4 \pm 0.9$ & $1.8 \pm 1.0$ & NS \\
\hline $\mathrm{FEV}_{1}(\mathrm{~L})$, mean $\pm \mathrm{SD}$ & $2.4 \pm 0.6$ & $2.9 \pm 0.9$ & NS \\
\hline
\end{tabular}

$\overline{A C Q-5,5-I t e m}$ Asthma Control Questionnaire; $A M$, morning; $C T$, computed tomography; $L M K$, Lund-Mackay; NS, not significant; UPSIT, University of Pennsylvania Smell Identification Test. 